\title{
Efficacy and safety of nanosomal docetaxel lipid suspension-based chemotherapy in squamous cell carcinoma of the head and neck: A multicenter retrospective study
}

\author{
SAROJ KUMAR DAS MAJUMDAR ${ }^{1}$, SUNDARAM SUBRAMANIAN ${ }^{2}$, GHANASHYAM BISWAS ${ }^{3}$, \\ NISARG JOSHI ${ }^{4}$, MUJTABA A. KHAN $^{4}$ and IMRAN AHMAD ${ }^{5}$ \\ ${ }^{1}$ Department of Radiotherapy, All India Institute of Medical Sciences, Bhubaneswar, Odisha 751019; \\ ${ }^{2}$ Department of Medical Oncology, VS Hospital, Madras Cancer Institute, Advanced Cancer Care, \\ Chennai, Tamil Nadu 600031; ${ }^{3}$ Department of Medical Oncology, Sparsh Hospital, Bhubaneswar, \\ Odisha 751007; ${ }^{4}$ Department of Medical Affairs and Clinical Development, Intas Pharmaceuticals Ltd., \\ Ahmedabad, Gujarat 380054, India; ${ }^{5}$ Jina Pharmaceuticals Inc., Libertyville, IL 60048, USA
}

Received March 25, 2020; Accepted September 10, 2020

DOI: $10.3892 / \mathrm{ol} .2020 .12207$

\begin{abstract}
Squamous cell carcinoma of the head and neck (SCCHN) is the most common cancer in Indian men. Docetaxel alone or in combination with other chemotherapeutic agents is recommended for the management of SCCHN. The present multicenter, retrospective study was conducted to evaluate the efficacy and safety of a novel docetaxel formulation 'nanosomal docetaxel lipid suspension (NDLS)'-based chemotherapy in SCCHN. The medical records of patients with SCCHN, who were treated with NDLS-based chemotherapy and followed up between August 2014 and September 2018, were reviewed. The efficacy endpoints were overall response rate [ORR; complete response $(\mathrm{CR})+$ partial response $(\mathrm{PR})]$ and disease control rate (DCR; $C R+P R+$ stable disease) for patients receiving NDLS-based induction or palliative chemotherapy. Overall survival (OS) and safety were also evaluated. Efficacy evaluation was available in 30/34 patients (induction, 20/23; palliative, 10/11). NDLS-based induction chemotherapy showed an ORR and DCR of $95 \%$ and a median OS of 43.5 months (follow-up duration, 0.6-80.3 months). For NDLS-based palliative chemotherapy, the ORR and DCR were $50 \%$ and the median OS time was 4.6 months (follow-up duration, 1.8 to 14.3 months). At least one adverse event was reported in $82.6 \%$ patients. No new safety concerns were reported. Overall, NDLS-based chemotherapy was effective and well tolerated in the treatment of SCCHN.
\end{abstract}

Correspondence to: Dr Imran Ahmad, Jina Pharmaceuticals Inc., 28100 N Ashley Circle, Suite 103, Libertyville, IL 60048, USA

E-mail: imran@jinapharma.com

Key words: DoceAqualip, nanosomal docetaxel lipid suspension, squamous cell carcinoma of the head and neck

\section{Introduction}

Squamous cell carcinoma of the head and neck (SCCHN) develops in the mucous membranes of the mouth, nose and throat, and can include carcinomas of the oral cavity, floor of the mouth, tongue, tonsils, juxtatonsillar fossae, larynx and pharynx (1). In India, lip and oral cavity cancer is the second most common cancer (119,992 cases; $11.54 \%)$ after breast cancer; it is the most common cancer among Indian men (92,011 cases; $16.1 \%)$ and the fourth most common cancer in Indian women (27,981 cases; 4.8\%) as per GLOBOCAN 2018 data (2). In total, $60-80 \%$ of SCCHN cases in India are diagnosed at advanced stages (3).

Early stage (I or II) SCCHN is usually treated with surgery and/or radiation therapy (RT), whereas multimodality treatment is generally required for patients with locally advanced (LA; stage III/IV) or metastatic SCCHN (4). The various treatment modalities include surgery followed by chemoradiotherapy (CRT), induction chemotherapy followed by CRT, RT or surgery (with or without adjuvant therapy), or epidermal growth factor receptor (EGFR) inhibition plus RT or CRT $(5,6)$.

Docetaxel in combination with cisplatin and 5-fluorouracil (5-FU), the TPF regimen, is approved for the induction therapy of patients with LA SCCHN (7). The TPF and docetaxel/cisplatin (TP) regimens are recommended as induction/sequential chemotherapy for SCCHN (6). Docetaxel plus cisplatin/carboplatin, docetaxel plus cisplatin/carboplatin plus cetuximab and docetaxel monotherapy are recommended for the treatment of recurrent, unresectable or metastatic SCCHN (6).

Polysorbate 80 and ethanol vehicles in the conventional docetaxel formulation can cause acute hypersensitivity reactions, peripheral neuropathy, cumulative fluid retention, reactions at infusion sites, severe anaphylactoid reactions and alcohol intoxication (8-12). Nanosomal docetaxel lipid suspension (NDLS; DoceAqualip; Intas Pharmaceuticals Ltd.), a novel lipid-based formulation, which is free from 
polysorbate 80 and ethanol, was developed to overcome these toxicity issues (13).

NDLS is approved in India for the induction treatment of LA SCCHN (14). Other approved indications for NDLS include the treatment of patients with androgen-independent (hormone refractory) metastatic prostate cancer, advanced gastric adenocarcinoma, locally advanced or metastatic breast cancer after failure of prior chemotherapy and non-small cell lung cancer after failure of prior chemotherapy (14). NDLS was developed using lipids generally regarded as safe by the US Food and Drug Administration based on the patented 'NanoAqualip' technology [patent number: Worldwide (WO2008127358), Europe (2076244), Japan (5917789) and Canada (CA2666322)] (15). The NDLS development process includes the addition of docetaxel to high-pressure homogenized soy phosphatidylcholine and sodium cholesteryl sulfate in sodium citrate buffer under continuous high-pressure homogenization (13), resulting in nanosomal $(<100 \mathrm{~nm})$ particles of NDLS (13). The delivery of docetaxel to tumor tissues is increased with these nanosomal particles, helped by the already damaged tumor vasculature, which results in an enhanced permeability and retention effect. A greater systemic availability of docetaxel (13) is seen, hence leading to improved therapeutic outcomes in terms of response rates (16).

NDLS has shown efficacy and safety in the treatment of breast, ovarian, cervical, penile, gastric, hormone refractory prostate, non-small cell lung, head and neck cancers, and sarcoma (14,16-22). The present study reports a real-world, multicenter, retrospective account of the use of NDLS-based chemotherapy in the treatment of SCCHN.

\section{Materials and methods}

Study design, patient selection and endpoints. The present study retrospectively reviewed the medical records of patients with SCCHN who were treated with NDLS-based chemotherapy as part of their clinical care and followed up between August 2014 and September 2018 at multiple centers including All India Institute of Medical Sciences, Bhubaneswar $(\mathrm{n}=10)$, VS Hospital, Madras Cancer Institute, Advanced Cancer Care, Chennai ( $\mathrm{n}=21)$, and Sparsh Hospital, Bhubaneswar $(n=3)$, India. The study inclusion criteria were: i) Patients of all age groups and ii) both sexes, with iii) histopathologically or cytologically confirmed tumors, and iv) patients who received NDLS as part of routine clinical practice, who had at least one measurable lesion as per the Response Evaluation Criteria in Solid Tumors (RECIST) 1.1 (23). Patients who had cancer other than SCCHN were excluded from this report. The efficacy endpoints included: i) Overall response rate (ORR), defined as the total proportion of patients achieving complete response (CR) plus those with a partial response (PR); ii) disease control rate (DCR), defined as the proportion of patients achieving $\mathrm{CR}+\mathrm{PR}+$ stable disease (SD); and iii) overall survival, defined as the time from treatment to death due to any cause. For patients who were still alive at the time of last follow-up (September 30, 2018) or who were lost to follow-up, OS was censored at the last recorded date that the patient was known to be alive. RECIST 1.1 was used for efficacy evaluation (23). Incidence of adverse events (AEs) were graded (where available) as per Common Terminology Criteria for Adverse Events 5.0 (24).

Ethics statement. The study was conducted after due approval from The OM Ethics Committee (Ahmedabad, India). The study was conducted in accordance with the ethical principles that have their origin in the Declaration of Helsinki (25), and in accordance with the International Conference on Harmonization's Good Clinical Practice guidelines (26), applicable regulatory requirements and in compliance with the submitted study protocol.

Statistical analysis. Demographic and baseline characteristics were summarized descriptively. Frequency and percentage were used for categorical variables and count, mean, standard deviation, median, minimum and maximum for continuous variables. The frequency and percentage of patients were used to present the response rates. Survival was analyzed using a non-parametric procedure performed using PROC LIFETEST (version 9.4; SAS Institute, Inc.). OS was measured using the Kaplan-Meier method and log-rank test. The AEs were summarized as frequencies and percentages by type of reaction.

\section{Results}

Patient disposition and demographics. In total, 228 patients with cancer who had received NDLS for their routine clinical care at different centers were evaluated. In the present report, the data of patients with SCCHN who received NDLS-based chemotherapy are presented.

Data of 34 patients with SCCHN, who were treated with NDLS-based chemotherapy regimens, were retrospectively analyzed. The baseline characteristics of these patients are summarized in Table I. The mean (SD) age of the patients was $54.70(10.2)$ years and majority $(73.52 \%)$ of the patients were men. Majority $(61.76 \%)$ of the patients had stage IV cancer. All the patients had the Eastern Cooperative Oncology Group (ECOG) scores (27) of either 0 or 1 . The most common anatomical sites for SCCHN were the mandible $(n=9)$, buccal mucosa $(n=7)$, hypopharynx $(n=5)$, tongue $(n=4)$, pharynx $(n=2)$, oral cavity, oropharynx, parotid $(n=1$ for each) and not specified $(n=4)$.

NDLS was administered as a 1-h infusion in 3-weekly cycles at $75 \mathrm{mg} / \mathrm{m}^{2}(\mathrm{n}=29 ; 85.2 \%)$ and $50 \mathrm{mg} / \mathrm{m}^{2}(\mathrm{n}=5 ; 14.8 \%)$; NDLS was used as first-line therapy in the majority $(91.2 \%)$ of the patients. Granulocyte-colony stimulating factor was used in all the patients as primary prophylaxis.

As induction chemotherapy $(n=23), 5$ patients received an NDLS-based TPF (NDLS, platinum and 5-FU) regimen, 6 received NDLS plus cisplatin, 9 received an NDLS-based TPF plus nimotuzumab regimen and 3 received an NDLS-based TPF plus cetuximab regimen. An NDLS-based TP regimen $(n=5)$, an NDLS-based TP plus nimotuzumab $(n=3)$, an NDLS-based TP plus cetuximab regimen $(n=2)$ and NDLS monotherapy $(n=1)$ were used as palliative chemotherapy $(n=11)$.

Efficacy. Of the 34 patients who received NDLS-based chemotherapy for the treatment of SCCHN as induction and 
Table I. Disposition and baseline characteristics of patients treated with induction $(n=23)$ and palliative $(n=11)$ chemotherapy.

\begin{tabular}{|c|c|c|c|}
\hline Parameters & All patients & Induction chemotherapy & Palliative chemotherapy \\
\hline Mean age \pm SD (range), years & $54.70 \pm 10.2(32-75)$ & $55.91 \pm 10.3(35-75)$ & $53.61 \pm 9.26(32-68)$ \\
\hline Mean $\mathrm{BSA} \pm \mathrm{SD}, \mathrm{kg} / \mathrm{m}^{2}$ & $1.56 \pm 0.2$ & $1.57 \pm 0.2$ & $1.55 \pm 0.22$ \\
\hline \multicolumn{4}{|l|}{ Sex, n $(\%)$} \\
\hline Male & $25(73.52)$ & $16(69.6)$ & $9(81.12)$ \\
\hline Female & $9(26.47)$ & $7(30.4)$ & $2(18.18)$ \\
\hline \multicolumn{4}{|l|}{ Cancer stage, n (\%) } \\
\hline I & $2(5.88)$ & $2(8.69)$ & - \\
\hline II & $5(14.70)$ & $5(21.74)$ & - \\
\hline III & $6(17.64)$ & $6(26.09)$ & - \\
\hline IV & $21(61.76)$ & $10(43.48)$ & $11(100.0)$ \\
\hline \multicolumn{4}{|l|}{ Metastasis site, $\mathrm{n}(\%)^{\mathrm{a}}$} \\
\hline Lymph node & $8(23.53)$ & $2(8.69)$ & $6(54.54)$ \\
\hline Lungs & $1(2.94)$ & - & $1(9.09)$ \\
\hline Liver & $1(2.94)$ & - & $1(9.09)$ \\
\hline Others & $1(2.94)$ & - & $1(9.09)$ \\
\hline \multicolumn{4}{|l|}{ ECOG performance score, n (\%) } \\
\hline 0 & $4(11.76)$ & $3(13.04)$ & $1(9.09)$ \\
\hline 1 & $30(88.23)$ & $20(86.96)$ & $10(90.91)$ \\
\hline \multicolumn{4}{|l|}{ Comorbid disease, n (\%) } \\
\hline Hypertension & $8(23.52)$ & $6(26.09)$ & $2(18.18)$ \\
\hline Diabetes & $3(8.82)$ & $3(13.04)$ & - \\
\hline Others $^{\mathrm{b}}$ & $6(17.64)$ & $1(4.35)$ & $5(45.45)$ \\
\hline
\end{tabular}

${ }^{\mathrm{a}}$ Metastasis site available for 9 patients only. ${ }^{\mathrm{b}}$ Other comorbid diseases included asthma, coronary artery disease, thrombophlebitis and dyslipidemia. BSA, body surface area; ECOG, Eastern Cooperative Oncology Group; SD, standard deviation.

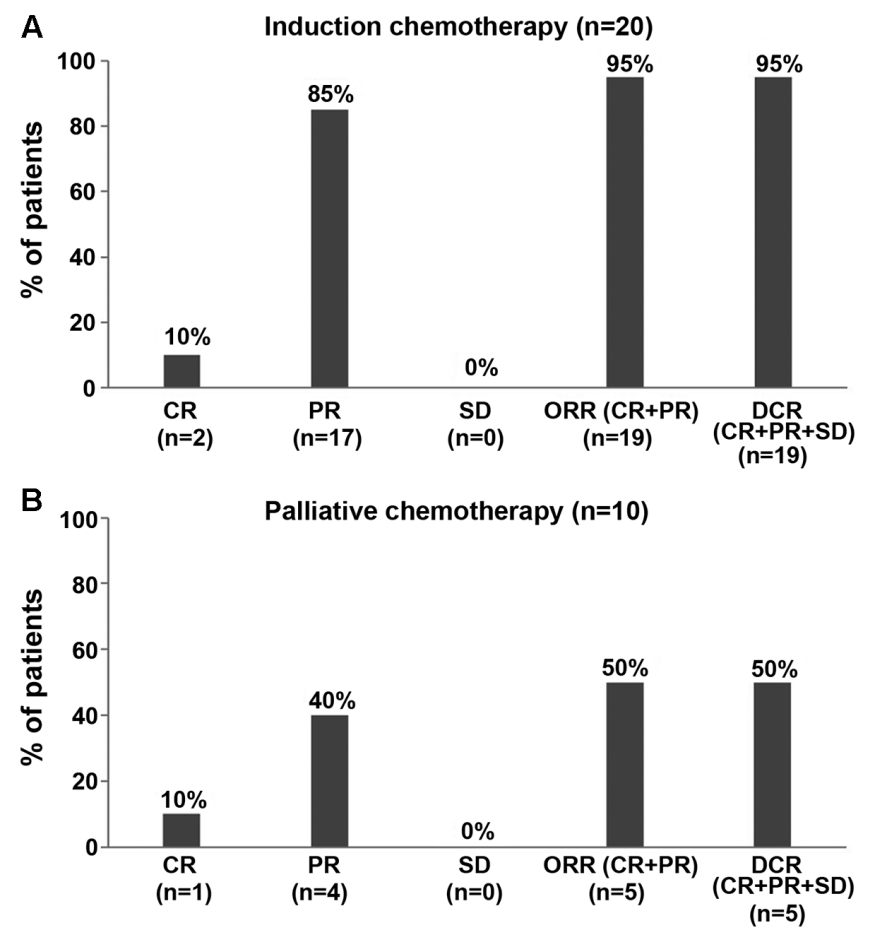

Figure 1. Efficacy of nanosomal docetaxel lipid suspension-based (A) induction $(\mathrm{n}=20)$ and $(\mathrm{B})$ palliative $(\mathrm{n}=10)$ chemotherapy for the treatment of squamous cell carcinoma of head and neck. Disease progression was observed in 5 patients who underwent palliative chemotherapy and 1 patient who underwent induction chemotherapy. CR, complete response; DCR, disease control rate; NDLS, nanosomal docetaxel lipid suspension; ORR, overall response rate; PR, partial response; $\mathrm{SD}$, stable disease. 
Table II. Response rates based on NDLS based chemotherapy regimens.

\begin{tabular}{lcc}
\hline Treatment regimen & No of patients treated & Response, $\mathrm{n}$ \\
\hline Induction chemotherapy (n=23) & & $\mathrm{PR}=3, \mathrm{PD}=1, \mathrm{NE}=1$ \\
NDLS-based TPF (NDLS, platinum and 5-FU) & 5 & $\mathrm{PR}=6$ \\
NDLS-based TP (NDLS plus cisplatin) & 6 & $\mathrm{CR}=2, \mathrm{PR}=6, \mathrm{NE}=1$ \\
NDLS-based TPF plus nimotuzumab & 9 & $\mathrm{PR}=2, \mathrm{NE}=1$ \\
NDLS-based TPF plus cetuximab & 3 & $\mathrm{CR}=1, \mathrm{PR}=1, \mathrm{PD}=3$ \\
Palliative chemotherapy ( $=11)$ & $\mathrm{PR}=2 . \mathrm{PD}=1$ \\
NDLS-based TP (NDLS plus cisplatin) & 5 & $\mathrm{PR}=1, \mathrm{PD}=1$ \\
NDLS-based TP plus nimotuzumab & 3 & $\mathrm{NE}=1$ \\
NDLS-based TP plus cetuximab regimen & 2 & 1 \\
NDLS monotherapy & 5 &
\end{tabular}

CR, complete response; NDLS, nanosomal docetaxel lipid suspension; NE, not evaluated; PR, partial response; PD, progressive disease; TP, taxane and platinum; TPF, taxane, platinum and 5-fluorouracil.
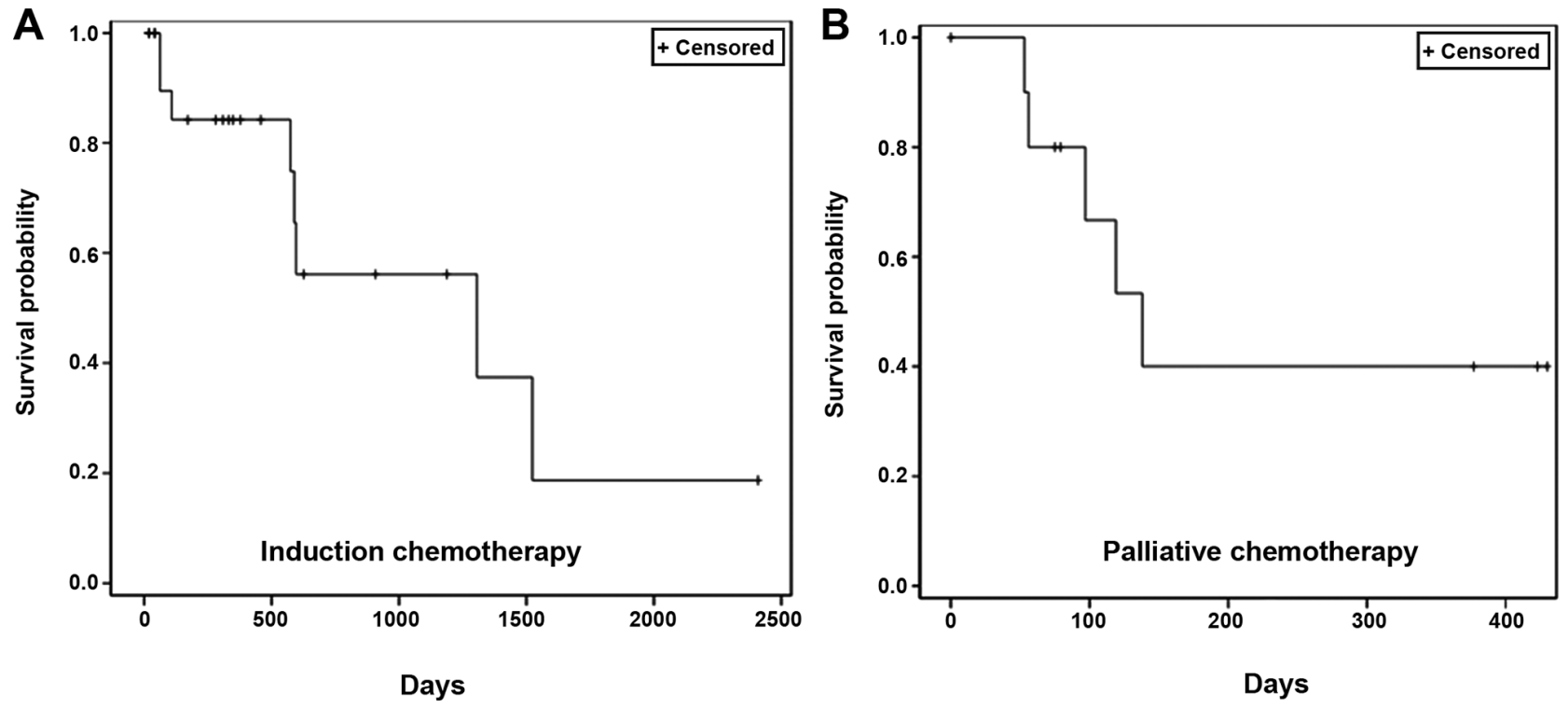

Figure 2. Kaplan Meier estimates of overall survival time in patients with squamous cell carcinoma of the head and neck treated with nanosomal docetaxel lipid suspension-based (A) induction $(\mathrm{n}=23)$ and $(\mathrm{B})$ palliative $(\mathrm{n}=11)$ chemotherapy.

palliative chemotherapy, an efficacy evaluation was possible for 30 patients (induction, 20/23 patients; palliative, 10/11 patients). The ORR and DCR were $95 \%$ each for NDLS-based induction chemotherapy $(\mathrm{CR}=10 \%, \mathrm{n}=2 ; \mathrm{PR}=85 \%, \mathrm{n}=17$; Fig. $1 \mathrm{~A}$; Table II) and 50\% each for NDLS-based palliative chemotherapy $(\mathrm{CR}=10 \%, \mathrm{n}=1 ; \mathrm{PR}=40 \%, \mathrm{n}=4$; Fig. 1B; Table II).

$O S$. The patient survival data were collected from the administration of the first dose of NDLS-based therapy until the date of last follow-up (September 30, 2018) for patients who were alive and until the date of death for patients who died. The proportion of patients who were alive at the last follow-up was $65.2 \%$ (15/23 patients) in the induction setting and $54.5 \%$ (6/11 patients) in the palliative setting. In the induction setting, the median OS was 43.5 months (follow-up duration, 0.6-80.3 months; Fig. 2A). In the palliative setting, the median OS was 4.6 months (follow-up duration, 1.8-14.3 months; Fig. 2B).
Safety. The data on AEs was available for 23 patients, and at least one $\mathrm{AE}$ was reported in $19(82.6 \%)$ of the patients. Grade 1 AEs were reported in $73.9 \%(17 / 23)$ patients, grade 2 in $13.0 \%(3 / 23)$ patients, grade 3 in $17.4 \%(4 / 23)$ patients and grade 4 in $4.3 \%$ (1/23) patients, respectively. Anemia, lymphopenia, thrombocytopenia and neutropenia were the reported hematological AEs, while hyperglycemia, constipation, nausea, vomiting and weakness were the most frequently reported non-hematological AEs (Table III). The grade 3/4 hematological AEs were neutropenia (8.7\%), lymphopenia (8.7\%) and thrombocytopenia $(4.3 \%)$. One $(4.34 \%)$ patient reported grade IV neutropenia.

\section{Discussion}

The treatment modality for LA SCCHN includes induction chemotherapy or CRT with a cisplatin and 5-FU combination as 
Table III. Safety profile of nanosomal docetaxel lipid suspension-based chemotherapy in squamous cell carcinoma of the head and neck $(n=23)$.

\begin{tabular}{lc} 
AEs & All grades, n $(\%)$ \\
\hline Hematological & \\
Anemia & $14(60.9)$ \\
Lymphopenia & $7(30.4)$ \\
Thrombocytopenia & $6(26.1)$ \\
Neutropenia & $3(13.04)$ \\
Non-hematological & \\
Hyperglycemia & $5(21.7)$ \\
Constipation & $2(8.7)$ \\
Nausea & $2(8.7)$ \\
Vomiting & $2(8.7)$ \\
Weakness & $2(8.7)$ \\
Anorexia & $1(4.3)$ \\
Diarrhea & $1(4.3)$ \\
Dyspnea & $1(4.3)$ \\
Hypotension & $1(4.3)$ \\
Mouth ulcer & $1(4.3)$ \\
Mucositis & $1(4.3)$ \\
Rash & $1(4.3)$
\end{tabular}

$\mathrm{AE}$, adverse event. AEs in different grades may occur in $\geq 1$ patient; hence, the cumulative number of patients in different grades may exceed the total number of patients with individual AEs.

the standard induction regimen (28). The addition of docetaxel to the standard TPF treatment as induction chemotherapy has shown significant survival benefits $(1,29)$. The TPF regimen is recommended for the induction/sequential chemotherapy of SCCHN by the European Head and Neck Society (EHNC), European Society for Medical Oncology (ESMO), and European SocieTy for Radiotherapy and Oncology (ESTRO) guidelines $(30,31)$. Furthermore, patients who achieve a CR or pathological CR after induction chemotherapy are likely to have a good prognosis (30).

An ORR of 68-87\% has been reported with docetaxel-based induction chemotherapy for the treatment of $\operatorname{SCCHN}(32,33)$, whereas in the present study, NDLS-based induction chemotherapy demonstrated an ORR of $95 \%$. The median OS with NDLS-based induction chemotherapy was 43.5 months (follow-up duration, 0.6-80.3 months). Docetaxel-based induction chemotherapy was previously evaluated in two phase III trials, TAX 323 (32) and TAX 324 (29). In the TAX 323 study, TPF induction chemotherapy $(n=177)$ resulted in an ORR of $68 \%$ and a median OS of 18.8 months (32). Similarly, TPF induction chemotherapy in the TAX 324 study $(n=255)$ resulted in an ORR of $72 \%$ and a median OS of 71 months (29). In a study by Pointreau et al (34), the TPF regimen $(\mathrm{n}=110)$ resulted in an ORR of $80 \%$. In the present study, the NDLS-based TPF regimen was used in 5 patients and resulted in PR in 3 ( $75 \%, 3$ out of 4 evaluated, $\mathrm{NE}=1$ excluded) of these patients. Previously, neoadjuvant chemotherapy with docetaxel and cisplatin $(n=34)$ demonstrated an ORR of $76.5 \%$ and a 3 -year
OS rate of $94.1 \%$ (35), compared with the $100 \%$ PR recorded in 6 patients (NDLS plus cisplatin) in the present study. Wang et al (33) used nimotuzumab, an anti-EGFR humanized monoclonal IgG1 antibody, in induction chemotherapy with the TPF regimen for LA SCCHN ( $n=31)$. This resulted in an ORR of $87.1 \%$ (30), compared with the $100 \%$ (CR, 2 patients; $\mathrm{PR}, 6$ patients; 8 out of 8 evaluated, $\mathrm{NE}=1$ excluded) recorded in the present study. In a phase III study, TPF induction chemotherapy followed by cetuximab showed a response rate of 78\% (36), while the same regimen was used in 3 patients in the present study with 2 patients achieving a PR (100\%, 2 out of 2 evaluated, $\mathrm{NE}=1$ excluded).

Docetaxel is recommended as a first-line therapy for recurrent, unresectable or metastatic SCCHN as a single agent or in combination with cisplatin/carboplatin with/without 5-FU/cetuximab (6). In this setting, docetaxel-based chemotherapy has reported an ORR of $33-97 \%$ in the treatment of SCCHN $(37,38)$. Patients receiving NDLS-based palliative chemotherapy demonstrated an ORR of $50 \%$ and a median OS of 4.6 months (follow-up duration, 1.8-14.3 months) in the present study. The Southwest Oncology Group evaluated the combination of docetaxel with carboplatin for advanced SCCHN $(n=68)$ and reported a response of $25 \%$ and a median OS of 7.4 months (39), while the NDLS-based TP regimen $(n=5)$ in the present study resulted in a CR and PR in 1 patient each.

In the conventional docetaxel formulation, polysorbate 80 and ethanol function as formulation vehicles, and have been implicated in AEs such as acute hypersensitivity reactions, cumulative fluid retention, peripheral neuropathy (8), severe non-immunological anaphylactoid reactions (9), reactions at injection sites (10) and alcohol intoxication $(11,12)$. In the present study, neurotoxicity, fluid retention and acute hypersensitivity reactions were not reported with NDLS-based chemotherapy.

In the landmark TAX 323 study, neutropenia (76.9\%), anemia $(9.2 \%)$, thrombocytopenia $(5.2 \%)$, febrile neutropenia $(5.2 \%)$ and leucopenia $(41.6 \%)$ were the grade $3 / 4$ hematological AEs, whereas alopecia (11.6\%), infections (6.9\%), stomatitis $(4.6 \%)$, lethargy $(2.9 \%)$, diarrhea $(2.9 \%)$, nausea, vomiting, neurotoxicity, anorexia and dysphagia (each $0.6 \%$ ) were the grade 3/4 non-hematological AEs following the TPF regimen. In the TAX 324 study, neutropenia (55\%), febrile neutropenia $(4.8 \%)$, anemia $(4.8 \%)$, thrombocytopenia $(1.6 \%)$ and neutropenic infections $(4.8 \%)$ were the grade $3 / 4$ hematological AEs. Meanwhile, stomatitis (8.4\%), nausea (5.6\%), dysphagia (5.2\%), anorexia (4.8\%), vomiting (3.2\%), diarrhea $(2.8 \%)$, infection $(2.4 \%)$ and lethargy $(2 \%)$ were the grade $3 / 4$ non-hematological AEs $(29,32)$. TPF was the most common regimen used in the present study, and neutropenia $(8.7 \%)$, lymphopenia $(8.7 \%)$ and thrombocytopenia $(4.3 \%)$ were the grade 3/4 hematological AEs observed. Grade 4 neutropenia was reported in $1(4.34 \%)$ patient. Vomiting and weakness were the most frequently reported non-hematological AEs. Overall, NDLS was found to be well tolerated in patients with SCCHN.

Corticosteroids are routinely administered as a premedication to mitigate the toxicity issues of conventional docetaxel, such as hypersensitivity and the retention of fluid (40). In a recent study, Obradović et al (41) used transcriptional profiling of tumors and matched metastases in patient-derived 
xenograft mouse models and indicated the potential function of glucocorticoid receptor activation in the progression and metastasis of breast cancer. Corticosteroid premedication is not warranted with the NDLS formulation, especially when used as monotherapy, therefore avoidance of corticosteroids may help circumvent the risk of disease progression.

There are several limitations to the present study. Due to the retrospective design, the data for safety and survival are incomplete. The information pertaining to the history of tobacco use was not available in the medical records of all patients and hence could not be presented. Progression-free survival data could not be obtained, as these data and serial scans were not available for the majority of patients at most of the follow-up time points.

Overall, the NDLS-based therapy was effective and well tolerated in the management of SCCHN either as induction or palliative chemotherapy. The present data provides valuable insights into the effectiveness and safety of NDLS in the management of SCCHN. A clinical study is currently underway to validate the present findings.

\section{Acknowledgements}

The authors would like to thank Mr. Shreekant Sharma, ISMPP $\mathrm{CMPP}^{\text {тм }}$ for providing assistance in writing the original manuscript and Dr Venugopal Madhusudhana, ISMPP CMPP for additional editorial assistance in the development of this manuscript (both Intas Pharmaceuticals Ltd., Ahmedabad, Gujarat, India).

\section{Funding}

This study was funded by an unrestricted research grant by Intas Pharmaceutical Ltd., Ahmedabad, Gujarat, India, towards data collection and statistical analysis.

\section{Availability of data and materials}

The datasets used and/or analyzed during the current study are available from the corresponding author on reasonable request.

\section{Authors' contributions}

SKDM, SS and GB performed the research, were involved in the acquisition of data and critically revised the manuscript for important intellectual content. SKDM, NJ, MAK and IA conceptualized and designed the study, and were involved in the data interpretation and critical revision of the manuscript for important intellectual content. All authors read and approved the final manuscript.

\section{Ethics approval and consent to participate}

The study protocol was reviewed and approved by The OM Ethics Committee (Ahmedabad, India). Dr Nisarg Joshi, who is an investigator and designed the concept of this multicenter retrospective study, is not attached to any institute having an ethics committee; hence, permission was sought from an independent ethics committee. The study was conducted in accordance with the ethical principles that have their origin in the Declaration of Helsinki, and in accordance with the International Conference on Harmonization's Good Clinical Practice guidelines, applicable regulatory requirements, and in compliance with the submitted study protocol. Patient consent to review their medical records was not required by the ethics committee as NDLS is already approved in India and patient confidentiality was completely maintained. In this retrospective study, no patient identifiers were used and patient data were anonymized.

\section{Patient consent for publication}

Not applicable.

\section{Competing interests}

Dr Mujtaba A. Khan and Dr Nisarg Joshi are employees of Intas Pharmaceutical Ltd (Ahmedabad, Gujarat, India). Dr Imran Ahmad is an employee of Jina Pharmaceutical Inc. (Libertyville, IL, USA). The NDLS (DoceAqualip) used in the present study was developed by Intas Pharmaceuticals Limited.

\section{References}

1. Rapidis A, Sarlis N, Lefebvre JL and Kies M: Docetaxel in the treatment of squamous cell carcinoma of the head and neck. Ther Clin Risk Manag 4: 865-886, 2008.

2. Bray F, Ferlay J, Soerjomataram I, Siegel RL, Torre LA and Jemal A: Global cancer statistics 2018: GLOBOCAN estimates of incidence and mortality worldwide for 36 cancers in 185 countries. CA Cancer J Clin 68: 394-424, 2018.

3. Kulkarni MR: Head and neck cancer burden in India. Int J Head Neck Surg 4: 29-35, 2013.

4. Machiels JP, Lambrecht M, Hanin FX, Duprez T, Gregoire V, Schmitz S and Hamoir M: Advances in the management of squamous cell carcinoma of the head and neck. F1000Prime Rep 6: 44, 2014.

5. Seiwert TY and Cohen EEW: State-of-the-art management of locally advanced head and neck cancer. Br J Cancer 92: 1341-1348, 2005.

6. NCCN Clinical Practice Guidelines in Oncology (NCCN Guidelines $\left.{ }^{\circledR}\right)$ : Head and Neck Cancer. Version 1.2019. https:// www.ncen.org/. Accessed March 14, 2019.

7. Van Cutsem E, Moiseyenko VM, Tjulandin S, Majlis A, Constenla M, Boni C, Rodrigues A, Fodor M, Chao Y, Voznyi E, et al: Phase III study of docetaxel and cisplatin plus fluorouracil compared with cisplatin and fluorouracil as first-line therapy for advanced gastric cancer: A report of the V325 study group. J Clin Oncol 24: 4991-4997, 2006.

8. ten Tije AJ, Verweij J, Loos WJ and Sparreboom A: Pharmacological effects of formulation vehicles: Implications for cancer chemotherapy. Clin Pharmacokinet 42: 665-685, 2003.

9. Coors EA, Seybold H, Merk HF and Mahler V: Polysorbate 80 in medical products and nonimmunologic anaphylactoid reactions. Ann Allergy Asthma Immunol 95: 593-599, 2005.

10. Schwartzberg LS and Navari RM: Safety of polysorbate 80 in the oncology setting. Adv Ther 35: 754-767, 2018.

11. Food and Drug Administration (FDA): FDA Drug Safety Communication: FDA warns that cancer drug docetaxel may cause symptoms of alcohol intoxication after treatment. https://www.fda. gov/Drugs/DrugSafety/ucm401752.htm. Accessed June 20, 2014.

12. Mirza A and Mithal N: Alcohol intoxication with the new formulation of docetaxel. Clin Oncol (R Coll Radiol) 23: 560-561, 2011.

13. Ahmad A, Sheikh S, Ali SM, Ahmad MU, Paithankar M, Saptarishi D, Maheshwari K, Kumar K, Singh J, Patel G, et al: Development of aqueous based formulation of docetaxel: Safety and pharmacokinetics in patients with advanced solid tumors. J Nanomed Nanotechnol 6: 1,2015.

14. Narayanan P, Dattatreya PS, Prasanna R, Subramanian S, Jain K, Somanath NS, Joshi N, Bunger D, Khan MA, Chaturvedi A and Ahmad I: Efficacy and safety of nanosomal docetaxel lipid suspension-based chemotherapy in sarcoma: A multicenter, retrospective study. Sarcoma 2019: 3158590, 2019. 
15. World Intellectual Property Organization: Aqueous systems for the preparation of lipid-based pharmaceutical compounds; compositions, methods, and uses thereof. Publication No. WO/2008/127358. https://patentscope.wipo. int/search/en/detail.jsf?docId=WO2008127358\&redirectedID = true. Accessed October 23, 2008.

16. Ahmad A, Sheikh S, Taran R, Srivastav SP, Prasad K, Rajappa SJ, Kumar V, Gopichand M, Paithankar M, Sharma M, et al: Therapeutic efficacy of a novel nanosomal docetaxel lipid suspension compared with taxotere in locally advanced or metastatic breast cancer patients. Clin Breast Cancer 14: 177-181, 2014.

17. Ashraf M, Sajjad R, Khan M, Shah M, Bhat Y and Wani Z: 156P Efficacy and safety of a novel nanosomal docetaxel lipid suspension (NDLS) as an anti cancer agent-a retrospective study. Ann Oncol 27 (Suppl 9): Six46-Six51, 2016.

18. Naik R and Khan MA: Doceaqualip in a patient with prostate cancer who had an allergic reaction to conventional docetaxel: A case report. Mol Clin Oncol 6: 341-343, 2017.

19. Prasanna R, Bunger D and Khan MA: Efficacy and safety of doceaqualip in a patient with locally advanced cervical cancer: A case report. Mol Clin Oncol 8: 296-299, 2018.

20. Vyas V, Joshi N and Khan M: Novel docetaxel formulation (NDLS) in low cardiac reserve ovarian cancer. Open Access J Cancer Oncol 2: 000122, 2018.

21. Gupta S, Pawar SS and Bunger D: Successful downstaging of locally recurrent penile squamous cell carcinoma with neoadjuvant nanosomal docetaxel lipid suspension (NDLS) based regimen followed by curative surgery. BMJ Case Rep 2017: bcr2017220686, 2017.

22. Murali A, Gupta S and Pendharkar D: Efficacy and tolerability of nanoparticle docetaxel lipid suspension. J Clin Oncol 36 (Suppl 15): e14542, 2018.

23. Eisenhauer EA, Therasse P, Bogaerts J, Schwartz LH, Sargent D, Ford R, Dancey J, Arbuck S, Gwyther S, Mooney M, et al: New response evaluation criteria in solid tumours: Revised RECIST guideline (version 1.1). Eur J Cancer 45: 228-247, 2009.

24. U.S. Department of Health and Human Services: Common Terminology Criteria for Adverse Events (CTCAE). Version 5.0. https://ctep.cancer.gov/protocoldevelopment/electronic_applications/docs/CTCAE_v5_Quick_Reference_5x7.pdf. Accessed November 27, 2017.

25. Dixon JR Jr: The international conference on harmonization good clinical practice guideline. Qual Assur 6: 65-74, 1998.

26. World Medical Association: World Medical Association Declaration of Helsinki: Ethical principles for medical research involving human subjects. JAMA 310: 2191-2194, 2013.

27. Datta SS, Ghosal N, Daruvala R, Chakraborty S, Shrimali RK, van Zanten C, Parry J, Agrawal S, Atreya S, Sinha S, et al: How do clinicians rate patient's performance status using the ECOG performance scale? A mixed-methods exploration of variability in decision-making in oncology. Ecancermedicalscience 13: 913, 2019.

28. Posner MR and Lefebvre JL: Docetaxel induction therapy in locally advanced squamous cell carcinoma of the head and neck. Br J Cancer 88: 11-17, 2003.

29. Posner MR, Hershock DM, Blajman CR, Mickiewicz E, Winquist E, Gorbounova V, Tjulandin S, Shin DM, Cullen K, Ervin TJ, et al: Cisplatin and fluorouracil alone or with docetaxel in head and neck cancer. N Engl J Med 357: $1705-1715,2007$.
30. Haddad R, Tishler R, Wirth L, Norris CM, Goguen L, Sullivan C, O'Donnell L, Li Y and Posner M: Rate of pathologic complete responses to docetaxel, cisplatin, and fluorouracil induction chemotherapy in patients with squamous cell carcinoma of the head and neck. Arch Otolaryngol Head Neck Surg 132: 678-681, 2006.

31. Grégoire V, Lefebvre JL, Licitra L, Felip E and EHNS-ESMO-ESTRO Guidelines Working Group: Squamous cell carcinoma of the head and neck: EHNS-ESMO-ESTRO clinical practice guidelines for diagnosis, treatment and follow-up. Ann Oncol 21 (Suppl 5): Sv184-Sv186, 2010.

32. Vermorken JB, Remenar E, van Herpen C, Gorlia T, Mesia R, Degardin M, Stewart JS, Jelic S, Betka J, Preiss JH, et al: Cisplatin, fluorouracil, and docetaxel in unresectable head and neck cancer. N Engl J Med 357: 1695-1704, 2007.

33. Wang X, Gu J, Shao C, Han K and Meng J: Nimotuzumab plus chemotherapy with docetaxel, cisplatin, 5-fluorouracil for locally advanced head and neck squamous cell carcinoma: A clinical study. J Cancer Res Ther 15: 312-316, 2019.

34. Pointreau Y, Garaud P, Chapet S, Sire C, Tuchais C, Tortochaux J, Faivre S, Guerrif S, Alfonsi M and Calais G: Randomized trial of induction chemotherapy with cisplatin and 5-fluorouracil with or without docetaxel for larynx preservation. J Natl Cancer Inst 101: 498-506, 2009

35. Hui EP, Ma BB, Leung SF, King AD, Mo F, Kam MK, Yu BK, Chiu SK, Kwan WH, Ho R, et al: Randomized phase II trial of concurrent cisplatin-radiotherapy with or without neoadjuvant docetaxel and cisplatin in advanced nasopharyngeal carcinoma. J Clin Oncol 27: 242-249, 2009.

36. Hitt R, Mesia R, Grau JJ, Iglesias L, Barco ED, Lozano A, Trufero JM, Giron CG, Martin AL and hernandez JJC: Randomized phase III trial of induction chemotherapy (ICT) with docetaxel-cisplatin-5fluorouracil (DCF) followed by cisplatin-radiotherapy (CRT) or cetuximab-radiotherapy (CetRT) in patients (pts) with locally advanced unresectable head and neck cancer (LAUHNC). J Clin Oncol 34 (Suppl 15): 6001, 2016.

37. Specht L, Larsen SK and Hansen HS: Phase II study of docetaxel and cisplatin in patients with recurrent or disseminated squamous-cell carcinoma of the head and neck. Ann Oncol 11: 845-849, 2000.

38. Rawat S, Tandan H, Patel S and Chaudhari S: Safety and efficacy of nimotuzumab with concurrent chemoradiotherapy in unresectable locally advanced squamous cell carcinoma of head and neck: An Indian rural hospital experience. South Asian J Cancer 8: 52-56, 2019.

39. Samlowski WE, Moon J, Kuebler JP, Nichols CR, Gandara DR, Ozer H, Williamson SK, Atkins JN, Schuller DE and Ensley JF: Evaluation of the combination of docetaxel/carboplatin in patients with metastatic or recurrent squamous cell carcinoma of the head and neck (SCCHN): A Southwest oncology group phase II study. Cancer Invest 25: 182-188, 2007.

40. Weiss RB, Donehower RC, Wiernik PH, Ohnuma T, Gralla RJ, Trump DL, Baker JR Jr, Van Echo DA, Von Hoff DD and Leyland-Jones B: Hypersensitivity reactions from taxol. J Clin Oncol 8: 1263-1268, 1990.

41. Obradović MMS, Hamelin B, Manevski N, Couto JP, Sethi A, Coissieux MM, Münst S, Okamoto R, Kohler H, Schmidt A and Bentires-Alj M: Glucocorticoids promote breast cancer metastasis. Nature 567: 540-544, 2019.

This work is licensed under a Creative Commons Attribution 4.0 International (CC BY 4.0) License. 